This item is the archived peer-reviewed author-version of:

\title{
Automatic forensic analysis of automotive paints using optical microscopy
}

\section{Reference:}

Thoonen Guy, Nys Bart, Vander Haeghen Yves, De Roy Gilbert, Scheunders Paul.- Automatic forensic analysis of automotive paints using optical microscopy

Forensic science international - ISSN 0379-0738 - 259(2016), p. 210-220

Full text (Publishers DOI): http://dx.doi.org/doi:10.1016/j.forsciint.2015.12.040

Handle/Permalink: http://hdl.handle.net/10067/1303530151162165141 


\title{
Automatic forensic analysis of automotive paints using optical microscopy
}

\begin{abstract}
The timely identification of vehicles involved in an accident, such as a hit-andrun situation, bears great importance in forensics. To this end, procedures have been defined for analyzing car paint samples that combine techniques such as visual analysis and Fourier Transform Infrared Spectroscopy. This work proposes a new methodology in order to automate the visual analysis using image retrieval. Specifically, color and texture information is extracted from a microscopic image of a recovered paint sample, and this information is then compared with the same features for a database of paint types, resulting in a shortlist of candidate paints. In order to demonstrate the operation of the methodology, a test database has been set up and two retrieval experiments have been performed. The first experiment quantifies the performance of the procedure for retrieving exact matches, while the second experiment emulates the real-life situation of paint samples that experience changes in color and texture over time.

Keywords: Automotive paint, Optical microscopy, Color calibration, Feature extraction, Image retrieval

2015 MSC: 00-01, 99-00
\end{abstract}

\section{Introduction}

In forensics, automotive paint fragments are one of the most commonly recovered traces of evidence at the scene of an accident, such as a hit-and-run scene [1]. As a logical consequence, linking these traces to a matching vehicle has 5 raised a considerable amount of attention. In recent years, multiple procedures 
have been defined that all aim to provide forensic experts with a well-defined, objective approach for characterizing the paint sample under study, which, in turn, can be used for identification. Such procedures often involve a combination of techniques, including macroscopic analysis, optical microscopy and spectroscopy, e.g. [2, 3]. Since automotive paints typically have a complex chemical composition, consisting of multiple layers (in essence, a primer, a color coat, and a clear coat finishing), techniques that assess this composition, such as microchemical analysis and X-Ray analysis as a non-destructive alternative, have been used extensively [4. Especially with the construction of the Paint Data Query (PDQ) database, i.e. a database with information ranging from physical attributes to an infrared (IR) spectrum for every layer of each of its more than 21000 paint samples [5], spectroscopy has found wide support. Techniques used for spectroscopic analysis include Fourier transform infrared spectroscopy (FTIR) and/or Raman spectroscopy [6, 7, 8]. Often these techniques are com${ }_{20}$ bined with pattern recognition methodologies, allowing automatic comparison of spectra [9, 10].

However, spectroscopy typically does not provide any information on the spatial heterogeneity of the paint sample under study. In addition, changes in chemical composition, e.g. due to migration of components between layers, 25 complicate classification [11, 12]. Consequently, visual analysis of the paint sample under study, assessing its color and its morphology, remains an important step in the identification of automotive paints. This visual analysis is routinely performed manually. Unfortunately, this technique is particularly prone to interoperator variability. Additionally, as time is of the essence in this particular type of police investigation, not only precise but also swift identification of the vehicle is of the uttermost importance. In this paper, we propose an objective and automatic approach for the visual analysis based on dark field microscopy. It is designed not only for the characterization, but also for the identification of automotive paint samples. The main aim of the new technique is to reduce the 35 workload of the experts and the backlog of cases considerably.

In order to fully characterize a sample, the proposed method assesses both 
the color distribution, and the texture of the sample. The assessment of the color distribution is a particularly challenging step, as it does not only require proper and reproducible lab practices, but also consistency of the images them-

40 selves. To this end, a color calibration procedure is defined, together with a newly developed reflective color chart for microscopy that contains only 20 test chart colors, in order to reduce the labor intensity of the procedure. After the calibration step, the color and texture are characterized by first separating the color from the texture. This separation is only possible after transformation of the image to an uncorrelated color space, such as the CIE $\mathrm{L}^{*} \mathrm{a}^{*} \mathrm{~b}^{*}$ space. Once this separation has been done, the color distribution is assessed by calculating the color histogram of the resulting color components. Likewise, texture features are extracted from the intensity component, in the form of Gabor features. Following the extraction of these features, suitable distance measures between 50 the image under test and each of the images in a database are selected with the aim of constructing a shortlist of matching images from this database. Such distance measures are selected for both the color histograms and for the Gabor features. Finally, in order to effectively create the aforementioned shortlist, both measures are combined in a sensible way, such that the matching images indeed reflect the highest visual resemblance to the image under test.

To validate the proposed approach, a retrieval experiment is performed in which the aim is to match several test paint samples with other instances of the same paint type in a database. Additionally, in order to demonstrate the robustness of our methodology against microscopic changes in visual appearance, as typically occurs because of wear and aging effects, a similar experiment is repeated using test paint samples that bear visual resemblance to certain database samples, but for which no instance of the same paint types is present in the database. For both of the aforementioned experiments, the effect of the color calibration step is demonstrated. 


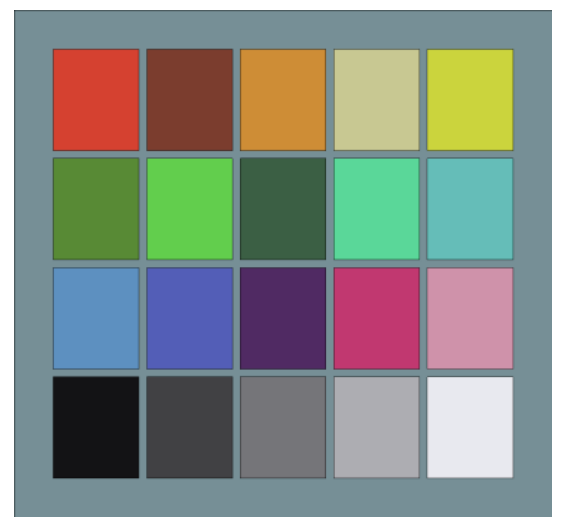

Figure 1: The calibration chart by DSC Labs [13. This chart has optimally placed colors for calibration.

\section{Material and methods}

\subsection{Color calibration}

Color calibration of acquired images plays an important role in the reproducible and accurate extraction of color features of an image. The color calibration transforms the unknown original image RGB data to a standardized RGB color space called sRGB [14, based on a set of color chart (see Fig. 1] images acquired together with the image being investigated under exactly the same circumstances and settings. Because the sRGB color space has a known relation to the CIE colorimetric spaces like CIE XYZ and CIE L*a* ${ }^{*}$ it is possible to compute color features in those spaces, and use perceptual color metrics like $\mathrm{dE}_{76}^{*}$ or $\mathrm{dE}_{2000}^{*}$ to express color differences. Although frequently encountered, it is simply incorrect to transform RGB data to e.g. CIE L*a* $\mathrm{b}^{*}$ without exact knowledge of the RGB primary colors (actually RGB is not a color space, but rather a family of color spaces which will be different, i.e. have different primaries, each time an image is acquired).

For details about the color calibration, see [15, 16, 17, but basically the color chart values as measured in the acquired images and their known colorimetric values as measured by a spectrophotometric device are used to construct a 3dimensional transform from the original image RGB data to the desired sRGB 

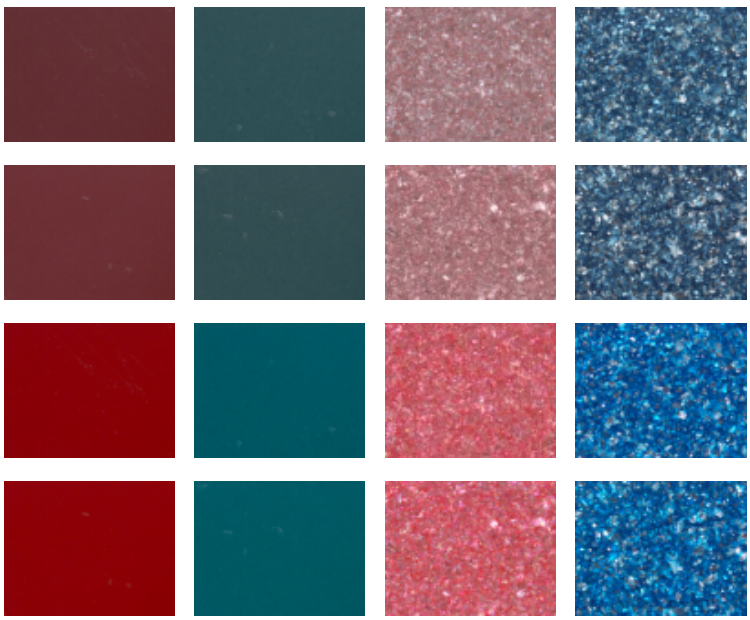

Figure 2: Top two rows: Uncalibrated images of two solid paints and two metallic paints under different microscope settings. Bottom two rows: Calibrated versions. Note how our acquisition application already removed a lot of variability due e.g. lighting.

data. This transform consists of three 1D lookup tables (LUT), combined with a 3D LUT, and the results for a set of test paint samples can be seen in Fig. 2, The camera used during the experiments was a Pixelink PL-A662 CMOS camera [18], with an Olympus BX60 microscope. The acquisition was performed using our own software in order to avoid any extraneous processing. This software also ensured proper exposure and white balance of the images using the white chart patch, thereby removing a lot of the variability inherent to normal microscope imaging. The median color difference between two measurements of the same sample in calibrated images is $1.3 \mathrm{dE}_{2000}^{*}$, with a maximum of $5 \mathrm{dE}_{2000}^{*}$. The median color difference of a color measurement with the spectrophotometric values is $1.1 \mathrm{dE}_{2000}^{*}$, also with a maximum of roughly $5 \mathrm{dE}_{2000}^{*}$.

\subsection{Feature extraction}

Conceptually, when considering a dark field microscopic image of an automotive paint sample, for example the one depicted in Fig 3(a), it is possible for a human interpreter to make a distinction between color and texture. In computer vision, on the other hand, this distinction is much less obvious when 


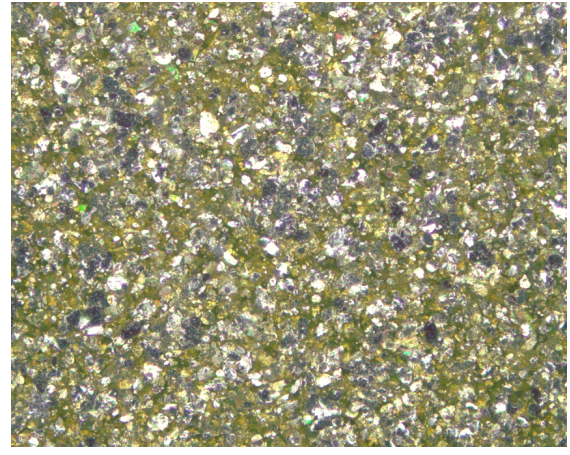

(a)

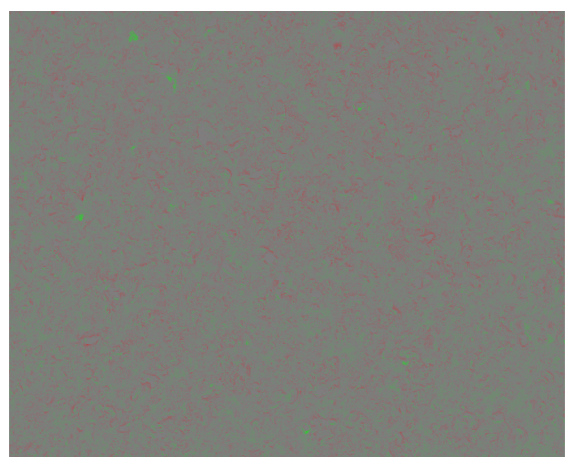

(c)

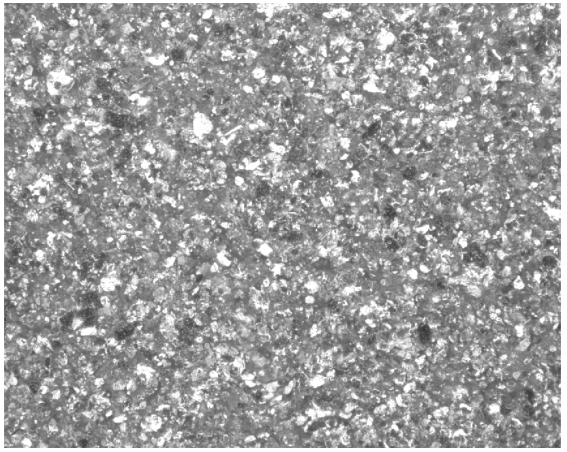

(b)

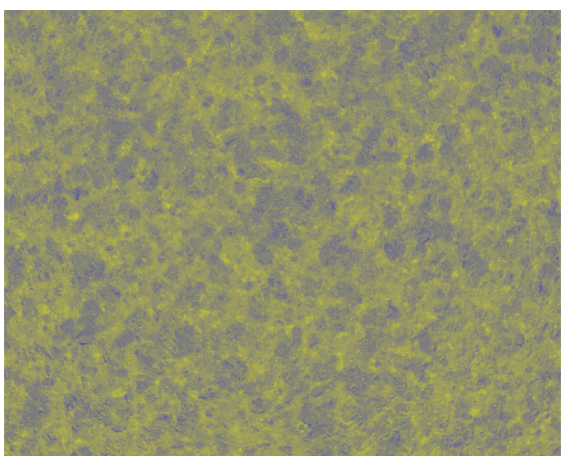

(d)

Figure 3: Illustration of the CIE $\mathrm{L}^{*} \mathrm{a}^{*} \mathrm{~b}^{*}$ representation of a dark field microscopic image of an automotive paint sample: (a) Original calibrated image (b) illumination component ' $\mathrm{L}$ ' (c) color component ' $a$ ' (opponent colors red and green) (d) color component 'b' (opponent colors yellow and blue).

working in an RGB color space, because all three color components, i.e. the red, blue and green intensity bands, respectively, are strongly correlated. A straightforward solution to this problem, is to transform the standardized sRGB color image into another, more suitable color space so we can treat color and texture components separately. One such color space is the CIE1976 L*a*b* space. In

$105 \mathrm{CIE} \mathrm{L}^{*} \mathrm{a}{ }^{*} \mathrm{~b}^{*}$ color space, the image is given by an illumination component ' $\mathrm{L}$ ', which is suitable for extracting texture information, and two color components ' $a$ ', which represents the opponent colors red and green, and 'b', which repre- 


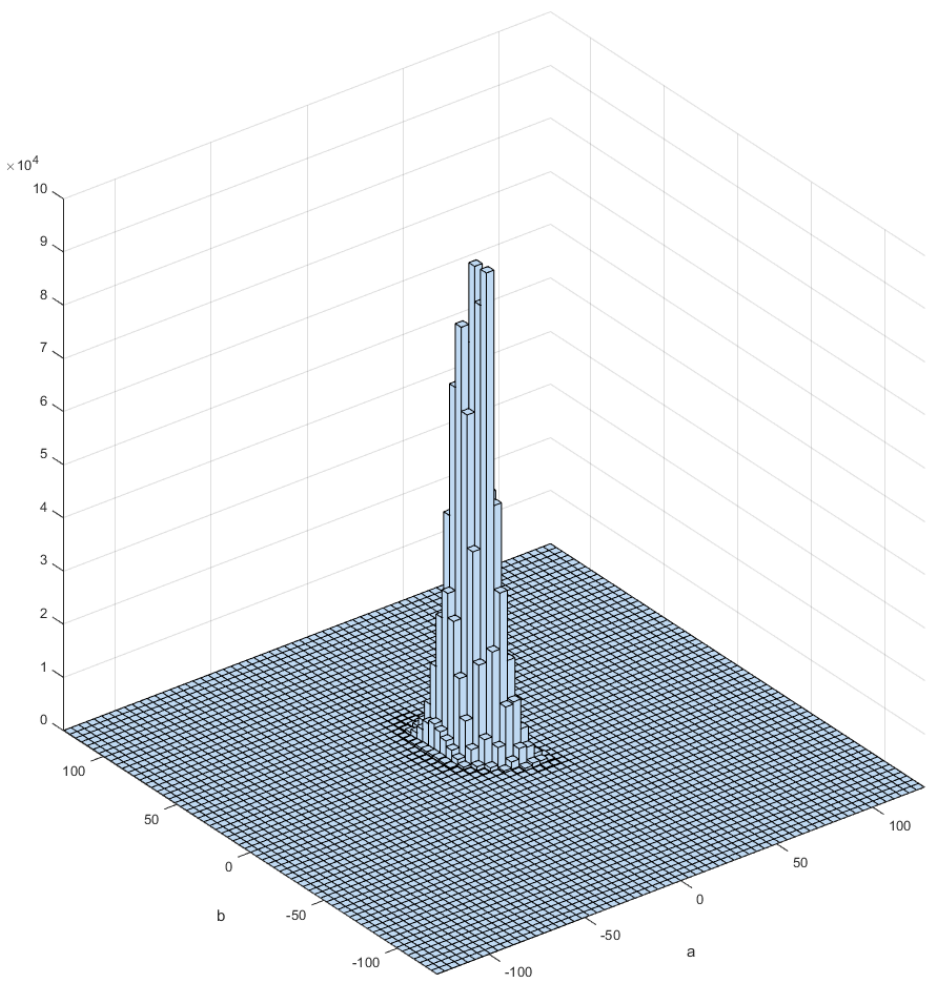

Figure 4: Illustration of the $2 \mathrm{D}$ color histogram for the automotive paint sample depicted in Fig. 3

sents the opponent colors yellow and blue [19. This concept is illustrated in Fig. 3(b), Fig. 3(c), and Fig. 3(d), respectively. Now that we have separated the color content from the texture, we can explore techniques for summarizing the color-based information and the texture information from the image, respectively. Let's first consider the color information. A simple methodology for estimating the color distribution from the image in CIE $\mathrm{L}^{*} \mathrm{a}^{*} \mathrm{~b}^{*}$ color space, is by calculating a $2 \mathrm{D}$ color histogram based on both the ' $\mathrm{a}$ ' and the ' $\mathrm{b}$ ' component. The color histogram for an image is constructed by counting the number of occurrences of pixels with a certain color in the image. In practice, such a histogram is generated by preliminary quantization of the intensity levels of each component into a number of bins, and then counting the number of image 
pixels in each bin. More formally,

$$
H_{I_{a}, I_{b}}\left(i_{a}, i_{b}\right)=N \cdot P\left(I_{a}=i_{a}, I_{b}=i_{b}\right)
$$

120

Here, $I_{a}$ and $I_{b}$ represent the intensity levels of the 'a' and 'b' color channels, and $N$ is the number of pixels in the image. Fig. 4 illustrates the 2D color histogram for the paint sample depicted in Fig. 3 .

The next step, after the extraction of the color features, is the extraction of textural information. In literature, many methodologies have been proposed for retrieving texture features, such as for instance the widely used Grey level Co-occurrence Matrix (GLCM) [20]. While this is a suitable approach for constructing a classification result on the level of individual pixels or objects, the goal of the current work is to summarize the texture features in an image with the aim of comparing them to the summarized texture features of other images. Hence, a desirable property of such texture features is that they provide both broad as well as local textural characteristics. This kind of information is obtained by using so-called multi-scale texture features. Many multi-scale approaches have been developed over the years (e.g. [21, 22]), but a common one is the use of Gabor filters [23.

A 2D Gabor filter is a filter for which the impulse response in the spatial domain is defined as the modulation of an harmonic function with a Gaussian,

$$
h(x, y)=\exp \left(-\frac{x^{\prime 2}+\gamma^{2} y^{\prime 2}}{2 \sigma^{2}}\right) \exp \left(j\left(\frac{2 \pi}{\lambda} x^{\prime}+\psi\right)\right)
$$

where $\sigma$ represents the standard deviation of the Gaussian, $\lambda$ and $\psi$ are the wavelength and the phase shift of the harmonic function, respectively, and $\gamma$ is the aspect ratio between $x^{\prime}$ and $y^{\prime}$. The spatial coördinates $x^{\prime}$ and $y^{\prime}$ are defined by the rotation of $x$ and $y$ over the angle $\phi$ as

$$
\left\{\begin{array}{l}
x^{\prime}=x \cos \phi+y \sin \phi \\
y^{\prime}=-x \sin \phi+y \cos \phi
\end{array}\right.
$$

Furthermore, a relationship exists between $\sigma$ and $\lambda$, which is parametrized by the so-called half amplitude bandwidth, as has been previously shown [24]. 


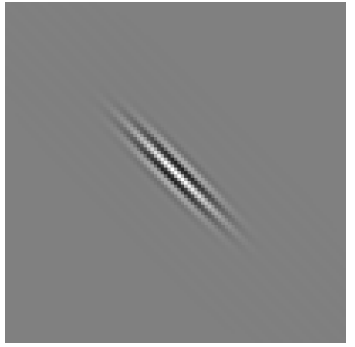

(a)

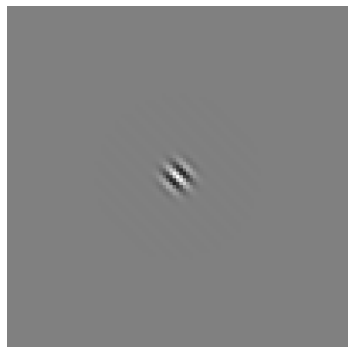

(d)

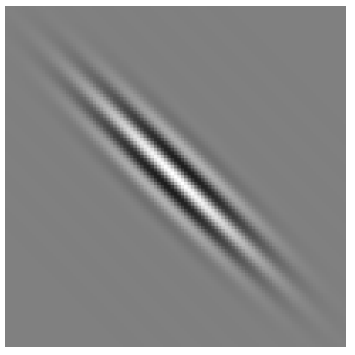

$(\mathrm{g})$

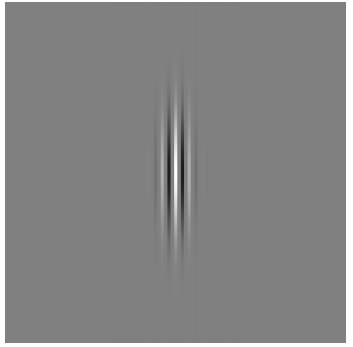

(b)

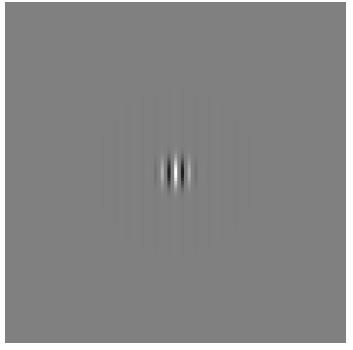

(e)

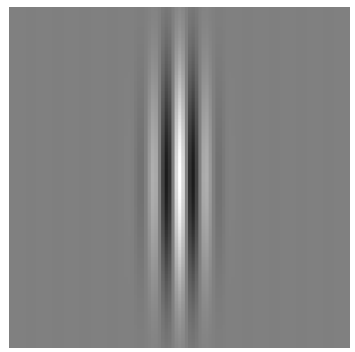

(h)

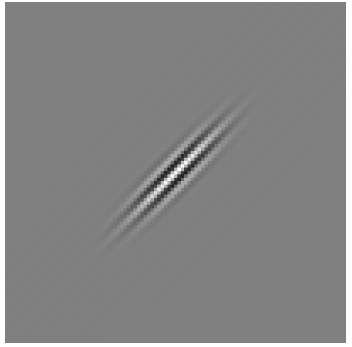

(c)

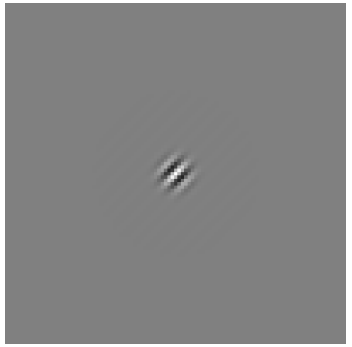

$(\mathrm{f})$

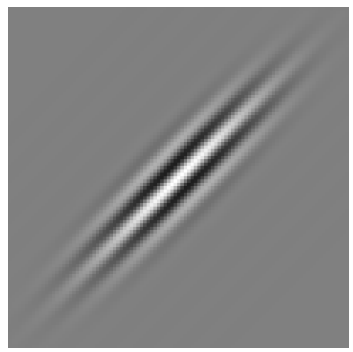

(i)

Figure 5: Illustration of the real part of the spatial impulse response function of the Gabor filter defined in Eq. 2 (a) Scale parameter $\lambda=4$, orientation parameter $\phi=\frac{\pi}{4}$, aspect ratio $\gamma=\frac{1}{4}$ (b) $\lambda=4, \phi=0, \gamma=\frac{1}{4}$ (c) $\lambda=4, \phi=-\frac{\pi}{4}, \gamma=\frac{1}{4}$ (d) $\lambda=4, \phi=\frac{\pi}{4}, \gamma=1$ (e) $\lambda=4$, $\phi=0, \gamma=1$ (f) $\lambda=4, \phi=-\frac{\pi}{4}, \gamma=1$ (g) $\lambda=8, \phi=\frac{\pi}{4}, \gamma=\frac{1}{4}$ (h) $\lambda=8, \phi=0, \gamma=\frac{1}{4}$ (i) $\lambda=8, \phi=-\frac{\pi}{4}, \gamma=\frac{1}{4}$.

For simplicity reasons, in this work, $\sigma=\lambda$ and the phase offset has been set to $\psi=0$. By varying the wavelength $\lambda$ (and therefore also $\sigma$ ) and the rotation angle $\phi$, a set of filters is constructed at various scales and orientations, respectively. This concept is illustrated in Fig. 5 for the real part of the Gabor 


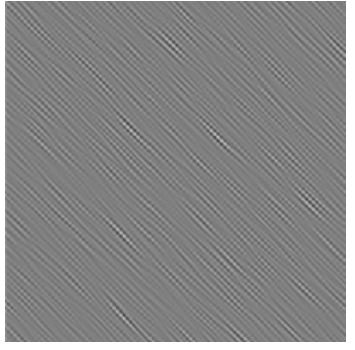

(a)

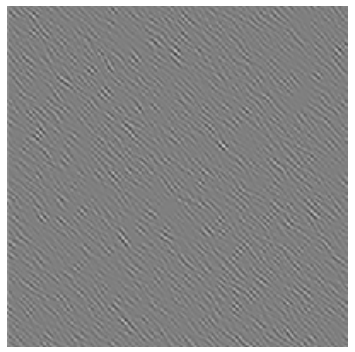

(d)

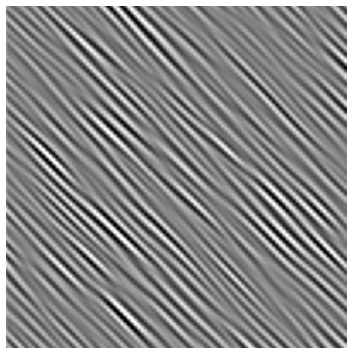

(g)

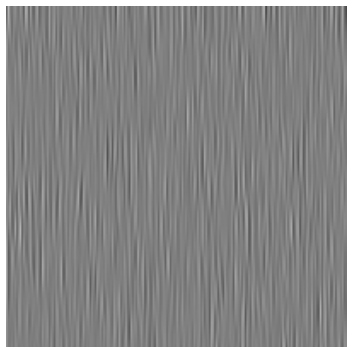

(b)

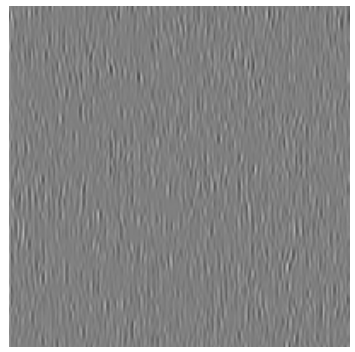

(e)

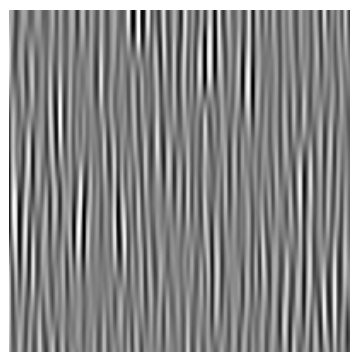

(h)

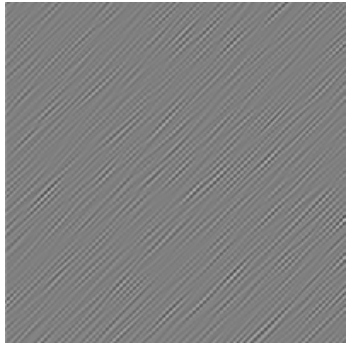

(c)

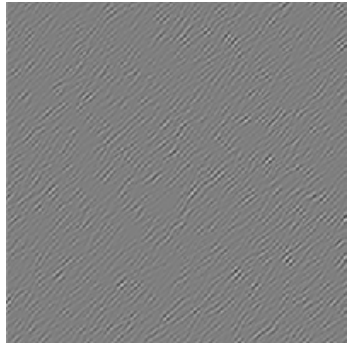

(f)

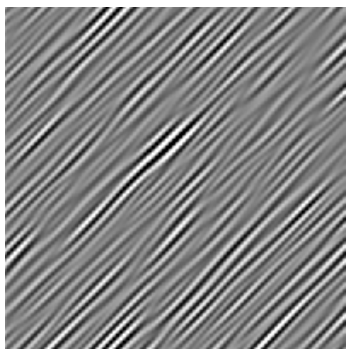

(i)

Figure 6: Illustration of the Gabor features obtained by applying the filters depicted in Fig. 5 on the car paint image shown in Fig. 3 (zoomed at a $200 \times 200$ pixels area): (a) Scale parameter $\lambda=4$, orientation parameter $\phi=\frac{\pi}{4}$, aspect ratio $\gamma=\frac{1}{4}$ (b) $\lambda=4, \phi=0, \gamma=\frac{1}{4}$ (c) $\lambda=4$, $\phi=-\frac{\pi}{4}, \gamma=\frac{1}{4}$ (d) $\lambda=4, \phi=\frac{\pi}{4}, \gamma=1$ (e) $\lambda=4, \phi=0, \gamma=1$ (f) $\lambda=4, \phi=-\frac{\pi}{4}, \gamma=1$ (g) $\lambda=8, \phi=\frac{\pi}{4}, \gamma=\frac{1}{4}$ (h) $\lambda=8, \phi=0, \gamma=\frac{1}{4}$ (i) $\lambda=8, \phi=-\frac{\pi}{4}, \gamma=\frac{1}{4}$.

filter defined in Eq. 2 using a few combinations of scale, orientation, and aspect ratio $\gamma$. As circular support is assumed, $\gamma$ is set to 1 in this work. Following the construction of the aforementioned set of filters, each of the corresponding impulse responses is convolved with the target image. The outcome of such a 
convolution is illustrated in Fig. 6, in which the result is shown of applying the filters in Fig. 5 to the car paint sample of Fig. 3. In this work, the textural content in an image for a certain $\lambda=m$ and $\phi=n$ is summarized by the mean $\mu_{m n}$ and the standard deviation $\sigma_{m n}$ of the Gabor coefficients. The resulting textural feature vector for a certain image is then simply the concatenation of these means and standard deviations for all the applied scales and orientations.

\subsection{Classification}

Once both color and texture features have been obtained for a database of automotive paint types and for the image under test, a distance measure is required for each feature type, as well as a way of combining the resulting distance measures, such that the matches can be ranked while taking into account all the feature types. As far as the $2 \mathrm{D}$ histograms representing the color features are concerned, a straightforward way is to do a simple bin-to-bin comparison by summing the differences between corresponding bins. Unfortunately, this approach tends to give more weight to relatively small differences between large bins while giving less weight to relatively large differences between small bins. A widely used solution to this problem is the Chi-Squared distance measure [25], which reduces the weight of large bins by normalizing the squared difference between two of these bins over the total number of elements in both bins. Given two images $I$ and $J$, the Chi-Squared distance between their histograms $H_{I_{a}, I_{b}}$ and $H_{J_{a}, J_{b}}$ is given by:

$$
d_{\text {color }}(I, J)=\chi^{2}\left(H_{I_{a}, I_{b}}, H_{J_{a}, J_{b}}\right)=\frac{1}{2} \sum_{i, j} \frac{\left(H_{I_{a}, I_{b}}(i, j)-H_{J_{a}, J_{b}}(i, j)\right)^{2}}{H_{I_{a}, I_{b}}(i, j)+H_{J_{a}, J_{b}}(i, j)}
$$

For the Gabor texture features, a similar reasoning is followed by applying a distance measure that reduces the impact of large differences between a textural feature if there is a high variation in this feature when considering all the images 175 in the database, and vice versa. Specifically, when comparing two images $I$ and $J$, the differences between the means $\mu_{m n}$ and the standard deviations $\sigma_{m n}$ of the Gabor features, with $m$ the scale and $n$ the orientation of the corresponding filter, are normalized over the standard deviation of the means $\alpha\left(\mu_{m n}\right)$ and of 
the standard deviations $\alpha\left(\sigma_{m n}\right)$ of all the database images, respectively [26]. ensuring focus for the complete imaged surfaces. Each of the lacquers, whether it's in the database or in the test set, bears a 7-digit color code identifier. In total, a database consisting of 221 paint samples has been measured, with color 
have been collected using the same camera setup. The setup itself consists of an Olympus BX60 microscope, equipped with a 10X/0.30 Bright field/Dark field UMPlanFL lens and a Nikon F1 camera. The imaged surface area under the experimental conditions used is $900 \times 600 \mu \mathrm{m}$.

210 ferred to as Subset 1) contains 14 samples of lacquer types of which other samples are present in the database. This randomly selected subset allows for a quantitative evaluation of the proposed method. The second subset (hereafter referred to as Subset 2) contains 8 samples that are not present in the database but that

sification has been performed combining color and texture features as described in section 2.3 .

\section{Results and Discussion}

\subsection{Subset 1}

In the first experiment, the proposed matching procedure is applied to test samples of lacquer types for which another sample is present in the database. This way, it is possible to find an exact match in the database, facilitating a quantitative evaluation of the approach. Once again, the experiment is con- 
Table 1: Shortlist ranking for each of the 14 test samples from Subset 1.

\begin{tabular}{|c||c|c|}
\hline \multirow{2}{*}{ Test sample color code } & \multicolumn{2}{|c|}{ Shortlist ranking } \\
\cline { 2 - 3 } & Before calibration & After calibration \\
\hline $6^{*} 08^{*} 04.80$ & 2 & 2 \\
$6^{*} 06^{*} 78.00$ & 1 & 1 \\
$5^{*} 10^{*} 08.50$ & 9 & 6 \\
$5^{*} 12^{*} 42.90$ & 11 & 12 \\
$5^{*} 12^{*} 47.00$ & 6 & 6 \\
$5^{*} 14^{*} 07.00$ & 1 & 1 \\
$5^{*} 16^{*} 21.00$ & 17 & 16 \\
$6 * 01 * 42.20$ & 4 & 2 \\
$6 * 02 * 40.00$ & 16 & 16 \\
$6 * 02 * 61.00$ & 4 & 4 \\
$6 * 03 * 21.20$ & 1 & 1 \\
$6 * 04 * 40.00$ & 4 & 3 \\
$6 * 05^{*} 12.30$ & 1 & 1 \\
$6 * 06 * 57.00$ & 1 & 1 \\
\hline
\end{tabular}

ducted both before and after color calibration. The results of this experiment are depicted in Table 1. The table contains the rank of the correct paint type in the shortlist. In other words, the lower the rank, the better the matching procedure succeeded in finding the corresponding paint type in the database. Once again, results before and after color calibration are quite close, with overall only slight improvements after color calibration. For each of the samples, both before and after color calibration, the correct match is found well within the first twenty candidates. Moreover, considering the results after color calibration, a positive match is found within the first six candidates for all but three test samples.

In the majority of samples from subset 1 , both feature types perform equally well, or with very small differences in performance. Hence, their combination yields very similar results. However, depending on the color and/or textural content of the image under test, situations do arise in which the combination 


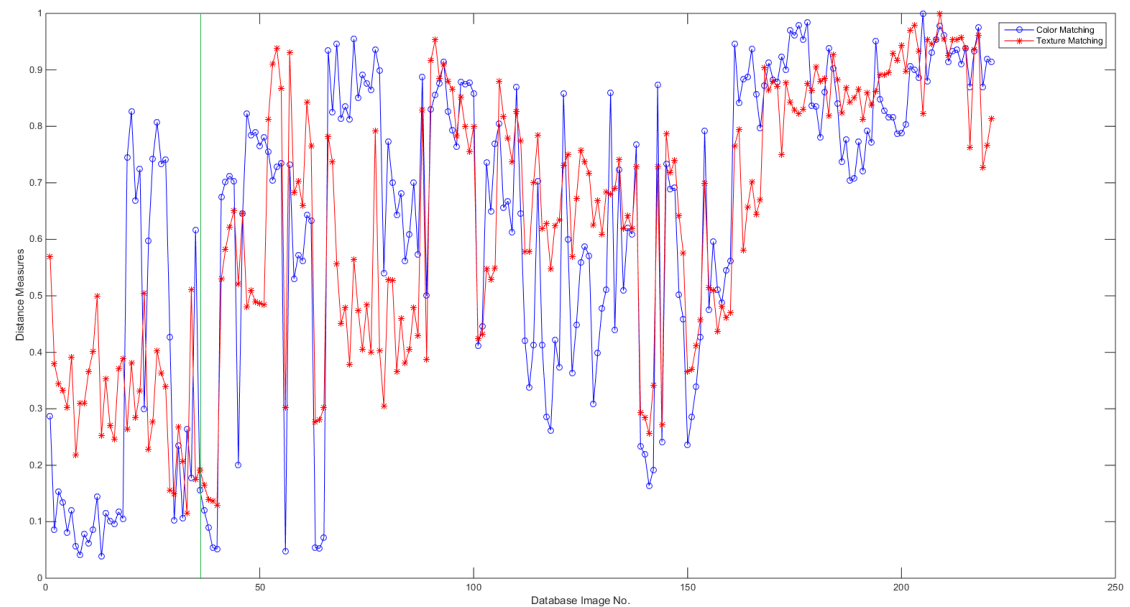

Figure 7: Normalized color and texture distance plots between the sample with color code $5 * 12 * 47.00$ and each of the database samples. The green vertical line indicates the correct match from the database.

of the features performs better than either one of the individual feature types. Fig. 7 illustrates how both features combined can result in a more accurate shortlist than when considering each of them separately. The figure shows a normalized distance graph for the sample under test with color code $5 * 12 * 47.00$. The correct match from the database is the sample for which the distances are indicated by the green vertical line. For this sample, if only the color features would be used, the rank of the correct match would be 28 . If only the texture features would be used, the rank of the correct match would be 9 . Combined, as already indicated in Table 1, the rank of the correct match is 6 .

\subsection{Subset 2}

These classification results show the outcome for test paint samples that are not present in the database but that do bear visual resemblance to database samples. For each feature type, the first three matches are displayed. Table 2 shows the outcome before color calibration. Visual resemblance of the candidate matches is very close to the sample under test, both in color distribution as in texture features. Table 3 depicts the outcome of the experiment after color 
Table 2: First three matches for the 8 test paint samples from Subset 2 before color calibration

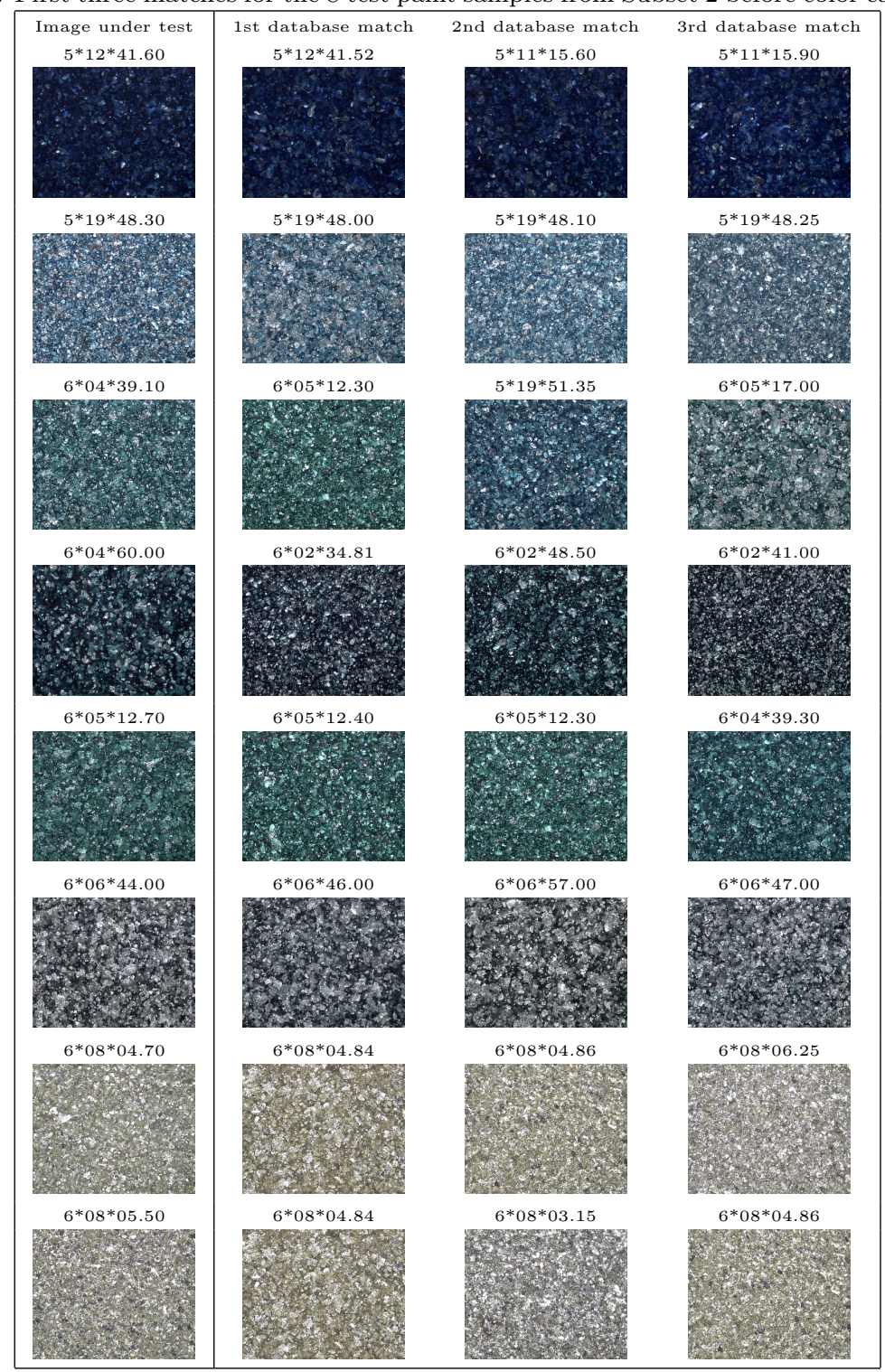




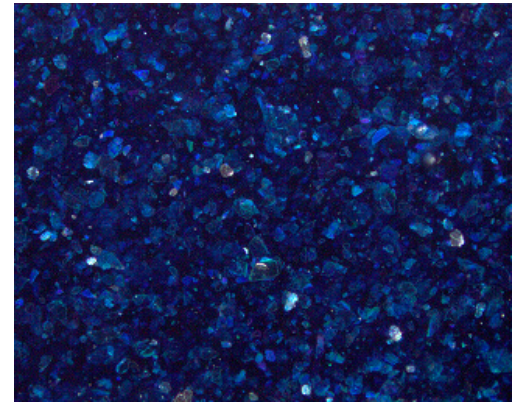

(a) $5^{*} 12^{*} 41.60$

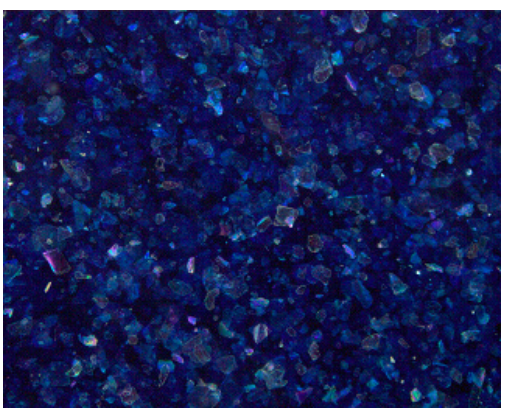

(c) $5 * 12.41 .52$

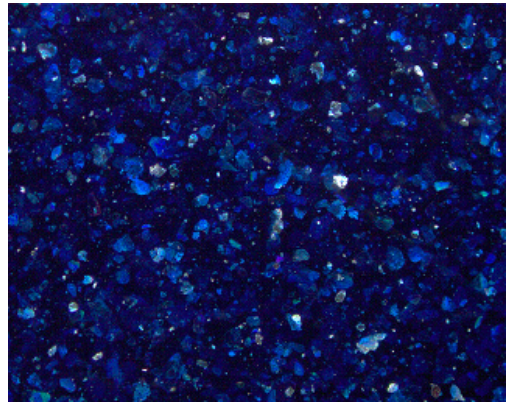

(b) $5 * 12 * 41.55$

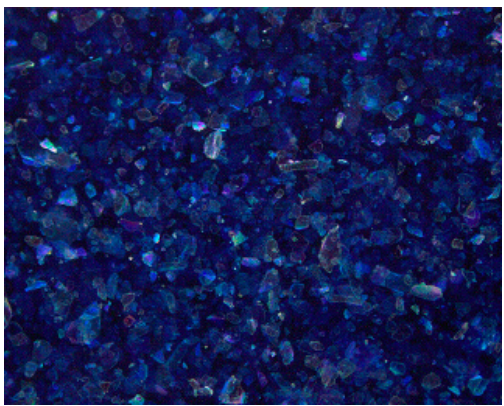

(d) $5 * 11 * 15.90$

Figure 8: Illustration of the discrepancy between color code and microscopic resemblance.

calibration. On visual comparison of the images of the test paint samples in the first column of Table 3 with their corresponding images in the first column of Table 2 , the effect of the color calibration on the appearance of the paint samples is very clear, showing an overall increase in contrast. Regarding classification, although in some cases the sequence of the first three matching samples has changed, almost no major changes occur in the result. Note, however, that this effect is to be expected, since the classification results already show a very high visual correspondence before color calibration. In other words, although visually the contrast clearly increases, the color calibration preserves prior resemblance.

The sample with color code $6 * 04^{*} 60.00$ is an excellent example of a paint sample for which no paint type is available in the database that matches both the color distribution and the textural features simultaneously. The first match 
Table 3: First three matches for the 8 test paint samples from Subset 2 after color calibration

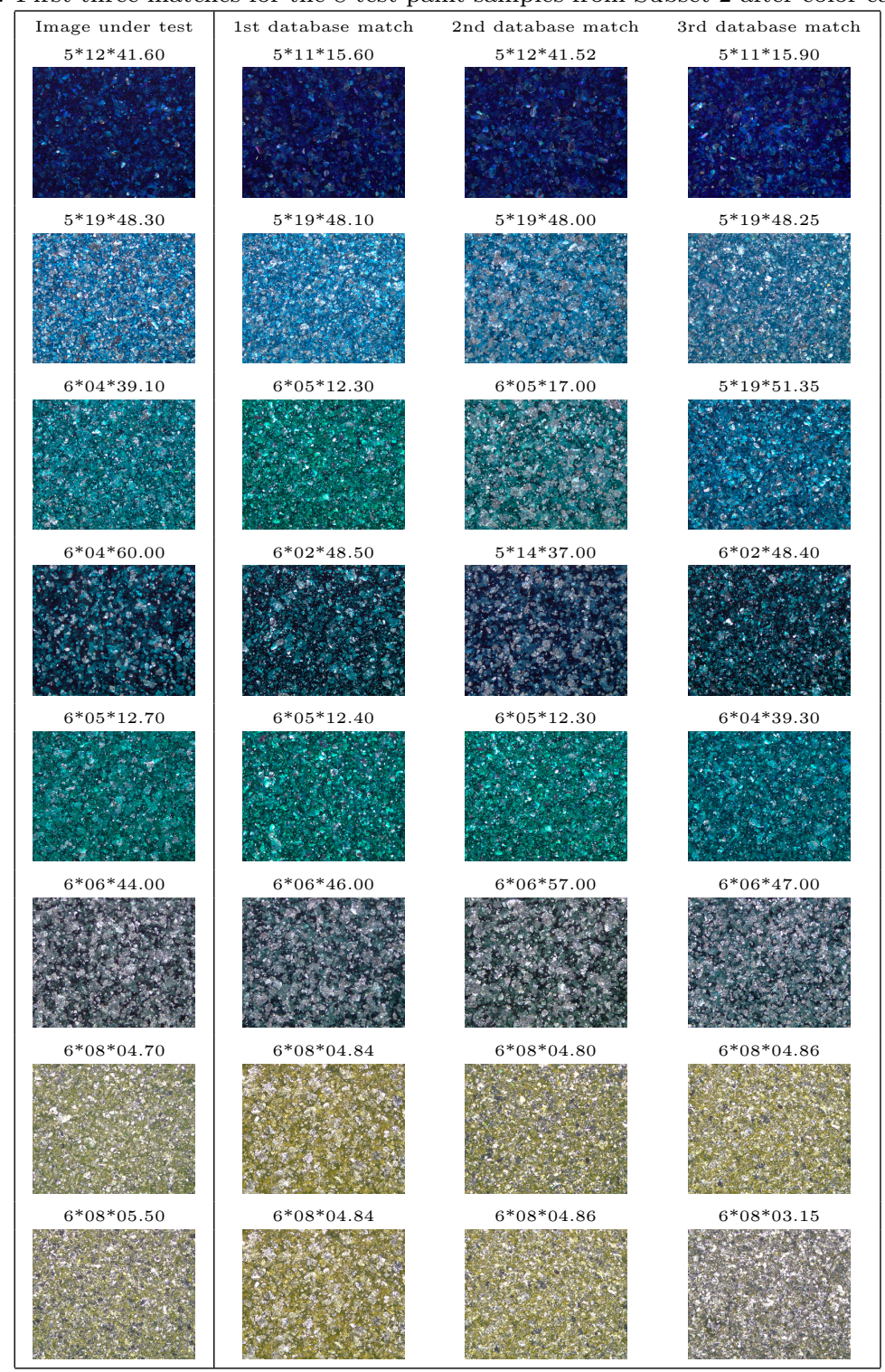


$(6 * 02 * 48.50)$ illustrates a better resemblance in color distribution than in texture, while the second match $\left(5^{*} 14^{*} 37.00\right)$ is an example of the opposite. Concerning the color code, often the code of the matching database samples is very close to the color code of the test sample under study. However, this correspondence is at most a weak indicator for the performance of the algorithm, as closeness in color code is not necessarily related to microscopic resemblance. This is illustrated in Fig. 8 for the test sample with color code $5^{*} 12^{*} 41.60$. While the paint sample with the closest color code in the database is $5^{*} 12^{*} 41.55$, it is obvious that the texture of this database sample is quite different from the test sample, which matches much more closely the appearance of $5^{*} 11^{*} 15.90$ and $5 * 12 * 41.52$.

\section{Conclusions}

In this article, a procedure is presented for automating the visual analysis of automotive paint samples as a valuable asset in the swift identification of vehicles in police investigations. The proposed methodology combines color calibration and the extraction of both color and texture information from microscopic images of paint samples that are collected at the scene of an accident. Subsequently, the extracted information is used to query a database of automotive paints in search for the best possible match. The result of the procedure is a shortlist of candidate paints suggested for further investigation. Experiments show that the proposed methodology is robust and shows promising results, both with paint samples for which a sample of the exact same lacquer type is present in the database, as for samples that bear resemblance to paint types in the database, the latter emulating real-life changes in color and texture that can occur over time.

[1] A. R. W. Jackson, J. M. Jackson, Forensic Science, 3rd Edition, Pearson Education Ltd, 2011.

¿ [2] ASTM E1610-14, Standard guide for forensic paint analysis and comparison, Standard, ASTM International, West Conshohocken, PA (2014). 
doi:10.1520/e1610

URL http: //www . astm.org

[3] SWGMAT, Guide for forensic paint analysis and comparison, Forensic Science Communications 1 (2).

[4] M. M. Houck, J. A. Siegel, Chapter 16 - paint analysis, in: Fundamentals of Forensic Science, 3rd Edition, Academic Press, San Diego, 2015, pp. 405-426. doi:10.1016/B978-0-12-800037-3.00016-9.

[5] B. K. Lavine, A. Fasasi, M. Sandercock, Improving PDQ database search strategies to enhance investigative lead information for automotive paints, Microchemical Journal 117 (2014) 133-137. doi:10.1016/j.microc. 2014.06 .007

[6] J. Zięba-Palus, R. Borusiewicz, Examination of multilayer paint coats by the use of infrared, Raman and XRF spectroscopy for forensic purposes, Journal of Molecular Structure 792-793 (2006) 286-292, 8th Polish Molecular Spectroscopy Conference. doi:10.1016/j.molstruc.2006.03.072

320 [7] J. Ziȩba-Palus, J. Wạs-Gubała, An investigation into the use of microraman spectroscopy for the analysis of car paints and single textile fibres,

【 Journal of Molecular Structure 993 (1-3) (2011) 127-133. doi:10.1016/ j.molstruc.2010.12.041.

[8] J. De Gelder, P. Vandenabeele, F. Govaert, L. Moens, Forensic analysis of automotive paints by Raman spectroscopy, Journal of Raman Spectroscopy 36 (11) (2005) 1059-1067. doi:10.1002/jrs.1408.

[9] A. Fasasi, N. Mirjankar, R.-I. Stoian, C. White, M. Allen, M. P. Sandercock, B. K. Lavine, Pattern recognition-assisted infrared library searching of automotive clear coats, Applied Spectroscopy 69 (1) (2015) 84-94. doi:doi:10.1366/14-07578.

[10] M. Maric, W. van Bronswijk, S. W. Lewis, K. Pitts, Synchrotron FTIR characterisation of automotive primer surfacer paint coatings for forensic 
purposes, Talanta 118 (2014) 156-161. doi:10.1016/j.talanta.2013. 10.016 .

[17] S. Van Poucke, Y. Vander Haeghen, K. Vissers, T. Meert, P. Jorens, Automatic colorimetric calibration of human wounds, BMC Medical Imaging 10 (1) (2010) 7. doi:10.1186/1471-2342-10-7.

[18] Pixelink.

[11] M. Maric, W. van Bronswijk, S. W. Lewis, K. Pitts, D. E. Martin, Characterisation of chemical component migration in automotive paint by synchrotron infrared imaging, Forensic Science International 228 (1-3) (2013) 165-169. doi:10.1016/j.forsciint.2013.01.032.

[12] K. J. van der Pal, G. Sauzier, M. Maric, W. van Bronswijk, K. Pitts, S. W. Lewis, The effect of environmental degradation on the characterisation of automotive clear coats by infrared spectroscopy, Talanta 148 (2016) 715720. doi:10.1016/j.talanta.2015.08.058.

[13] DSC Labs,

URL http://dsclabs.com/

[14] IEC 61966-2-1, Multimedia systems and equipment - Colour measurements and management - Part 2-1: Colour management - Default RGB color space - sRGB (International Electrotechnical Commission, Geneva, 199910) (1999).

[15] Y. Vander Haeghen, J. Naeyaert, I. Lemahieu, W. Philips, An imaging system with calibrated color image acquisition for use in dermatology, Medical Imaging, IEEE Transactions on 19 (7) (2000) 722-730. doi:10.1109/42.875195,

[16] Y. Vander Haeghen, J. Naeyaert, Consistent cutaneous imaging with commercial digital cameras, Archives of Dermatology 142 (1) (2006) 42-46. doi:10.1001/archderm.142.1.42.
URL http://www.pixelink.com/ 
[19] J. Schanda (Ed.), Colorimetry: Understanding the CIE System, John Wiley \& Sons, Inc., 2007. doi:10.1002/9780470175637.

[20] R. M. Haralick, K. Shanmugam, I. Dinstein, Textural features for image classification, IEEE Transactions on Systems, Man, and Cybernetics SMC3 (6) (1973) 610-621. doi:10.1109/TSMC. 1973.4309314

[21] T. Celik, T. Tjahjadi, Multiscale texture classification using dual-tree complex wavelet transform, Pattern Recognition Letters 30 (3) (2009) 331-339. doi:10.1016/j.patrec.2008.10.006

[22] K.-O. Cheng, N.-F. Law, W.-C. Siu, Multiscale directional filter bank with applications to structured and random texture retrieval, Pattern Recognition 40 (4) (2007) 1182-1194. doi:10.1016/j.patcog.2006.07.014

[23] M. Clark, A. C. Bovik, W. S. Geisler, Texture segmentation using Gabor modulation/demodulation, Pattern Recognition Letters 6 (4) (1987) 261267. doi:10.1016/0167-8655(87)90086-9.

375 [24] T. S. Lee, Image representation using 2d gabor wavelets, Pattern Analysis and Machine Intelligence, IEEE Transactions on 18 (10) (1996) 959-971. doi:10.1109/34.541406.

[25] O. Pele, M. Werman, The quadratic-Chi histogram distance family, in: K. Daniilidis, P. Maragos, N. Paragios (Eds.), Computer Vision - ECCV 2010, Vol. 6312 of Lecture Notes in Computer Science, Springer Berlin Heidelberg, 2010, pp. 749-762. doi:10.1007/978-3-642-15552-9_54.

[26] B. Manjunath, W. Ma, Texture features for browsing and retrieval of image data, Pattern Analysis and Machine Intelligence, IEEE Transactions on 18 (8) (1996) 837-842. doi:10.1109/34.531803.

${ }_{385}$ [27] M. Ehrgott, M. M. Wiecek, Mutiobjective programming, in: J. Figueira, S. Greco, M. Ehrgott (Eds.), Multiple Criteria Decision Analysis: State of the Art Surveys, Vol. 78 of International Series in Operations Research 
\& Management Science, Springer New York, 2005, pp. 667-708. doi:10.

1007/0-387-23081-5_17. 